\begin{tabular}{|c|c|c|}
\hline Beitr. Ent. & Keltern & ISSN 0005-805X \\
\hline $\mathbf{6 1}(2011) 2$ & S. $277-284$ & 10.11 .2011 \\
\hline
\end{tabular}

\title{
Beitrag zur Kenntnis der Lepidoptera-Fauna des Sudan
}

\section{(Lepidoptera, Pterophoridae)}

\section{Ernst Arenberger, Vladimir Gurko und Samson A. Binyason}

Mit 11 Figuren

\section{Summary}

Three species of the family Pterophoridae are recorded as new for the fauna of the Sudan. Two species of them are new for science: Hellinsia planiunci sp. n. and Paulianilus lolibai sp. n. The genitalia and adults of all species are depicted.

Key words

Lepidoptera, Pterophoridae, Hellinsia planiunci sp. n., Paulianilus lolibai sp. n., Sudan.

\section{Zusammenfassung}

Es werden drei Arten der Familie Pterophoridae als neu für die Fauna des Sudan gemeldet. Davon sind zwei Arten auch neu für die Wissenschaft: Hellinsia planiunci sp. n. und Paulianilus lolibai sp. n. Alle Arten werden abgebildet.

\section{Einleitung}

Die vorliegende Publikation kam durch das Zusammenwirken glücklicher Umstände zustande, die es dem Zweitautor ermöglicht hatten, durch die Mithilfe der offiziellen Stellen des Sudan eine Sammelreise in den Südsudan durchzuführen. Als Resultat liegt nun eine kleine Ausbeute von Pterophoridae vor. Die Aufsammlungen wurden im Süd-Sudan, in den Lolibai Mountains, gemacht (Fig. 1).

\footnotetext{
Abkürzung der Sammlungen

NHMW N Naturhistorisches Museum Wien
}

Pterophorus albidus (Zeller, 1852) (Fig. 2, 5-6)

Aciptilus albidus ZeLLER, Linnea Entomologica 6: 397. Locus typicus: Südafrika.

Material:

$2 \sigma^{\star} \sigma^{\star}$ : Southern Sudan, East Equatorial State, Akotos province, Lolibai Mts., $1300 \mathrm{~m}, 15.8$.10.9.2010, leg. V. Gurko. GU 5878 ð Ar., Coll. Arenberger (NHMW). 


\section{Verbreitung:}

Sudan, Tropisches Afrika, Madagaskar, Vietnam, Indonesien, Thailand, Japan, Neue Hebriden, Fidschi, Salomonen, Neuguinea, Australien.

\section{Erstmeldung für den Sudan.}

\section{Hellinsia planiunci sp. n. (Fig. 3)}

Holotypus, ơ: Southern Sudan, East Equatorial State, Akotos province, Lolibai Mts., 1300 m, 15.8.-10.9.2010, leg. V. Gurko. GU 5509 ๙ Ar. Coll. Arenberger (NHMW).

Paratypen: $12 \sigma^{\star} \sigma^{\star}, 2$ ㅇ q (GU 5506, 5507, $5512 \sigma^{\star}, 5511$ ㅇ) mit den gleichen Daten wie der Holotypus. Coll. Arenberger (NHMW).

\section{Etymologie:}

planus (lat.): eben, flach, platt; Uncus: Teil des männlichen Genitals.

\section{Diagnose:}

Expansion 16-17 mm. Vorderflügel etwa zu 1/3 gespalten, gelblichweiß. Fransen ebenfalls gelblichweiß, Innenrandfransen des Spaltenraumes des Vorderzipfels geschwärzt. Am Innenrand des Hinterzipfels knapp vor der Spitze mit einem schwarzen Wisch. Hinterflügel einfarbig gelblichweiß.

Genitalien, ${ }^{\rightarrow}$ (Fig. 7-8):

Linke Valve breiter als rechte, mit einem langen, zum Innenrand gebogenen Sacculusfortsatz; rechte Valve nur mit einer kurzen Sklerotisierung im Mittelteil; Uncus auffallend breit, plattenförmig, an seiner Spitze mit einem kurzen Dorn; beide Anellusarme gleichförmig, relativ lang. Aedoeagus leicht gebogen, in eine Spitze endend.

Genitalien, + (Fig. 11):

Antrum stark sklerotisiert, zur rechten Pleurawand hin abgebogen, Caudalende gabelförmig. Über dem Ostium befindet sich eine halbkreisförmige Chitinspange; Ductus bursae etwa die Bursalänge messend, letztere sackförmig; Apophyses posteriores relativ derb, etwa gleich lang wie die Papillae anales.

\section{Erste Stände und Ökologie:}

Flugzeit VIII - IX. Flughöhe: 1300 m. Futterpflanze unbekannt.

\section{Artabgrenzung:}

Der linke Sacculusfortsatz des männlichen Genitals ähnelt sehr dem von Merrifieldia innae Kovtunovich \& Ustjuzhanin, 2011 (im Druck), die aus Lesotho beschrieben wird. $H$. planiunci ist jedoch neben anderen Merkmalen vor allem durch den breiten, plattenförmigen Uncus von dieser unterschieden. 
Paulianilus lolibai sp. n. (Fig. 4)

Holotypus, ơ: Southern Sudan, East Equatorial State, Akotos province, Lolibai Mts., $1300 \mathrm{~m}$, 15.8.-10.9.2010, leg. V. Gurko. GU 5510 o Ar. Coll. Arenberger (NHMW).

Paratypus, $10^{\star}$ : mit den gleichen Daten wie der Holotypus. Coll. Arenberger (NHMW).

\section{Etymologie:}

Lolibai Mts. - ein Gebirgszug im Südsudan.

\section{Diagnose:}

Expansion $15 \mathrm{~mm}$. Vorderflügel mittelbraun, mit zahlreichen dunkelbraunen Schuppen übergossen. Einschnitt des Vorderflügels 2/6 der Flügellänge messend. An der Spaltung und knapp davor mit je einem schwarzen Punkt, sowie einem Pünktchen am Costalrand bei etwa halber Länge des Vorderzipfels und einem weiteren knapp danach. Fransen im Spaltenraum ockerfarben, an den Zipfelenden dunkelbraun. Basen der Innenrandfransen entlang des Hinterzipfels ockerfarbig, deren Spitzen dunkelbraun. Hinterflügel einfarbig braun.

Genitalien, ơ (Fig. 9-10):

Linke Valve bedeutend voluminöser als rechte; Sacculusfortsatz der rechten Valve nur aus einem auswärts gebogenen, spitz endenden Zapfen bestehend. Die linke hingegen mit einem spitzen Dorn knapp nach der Sacculusbasis und, entlang des Innenrandes weiterlaufend, mit einem langen, bis 4/5 der Valvenlänge messsenden Arm. An der Valvenbasis mit einem kräftigen Sporn. Rechter Anellusarm etwas länger als linker. Uncus spitz, dornenförmig. Aedoeagus nach der halben Länge leicht abgebogen, spitz endend.

\section{Erste Stände und Ökologie:}

Flugzeit VIII - IX. Höhenlage 1300 m. Futterpflanze unbekannt.

\section{Bemerkung:}

Das vorliegende Material wurde mit den Abbildungen der Paulianilus - Arten bei Gibeaux, 1994 verglichen und es handelt sich mit größter Wahrscheinlichkeit um eine Art dieser Gattung, die P. madecasseus (Вigot, 1964) sehr nahe steht. Doch fehlt dieser der basale Sporn der linken Valve.

\section{Danksagung}

Für die bei der Vorbereitung und Durchführung unserer Expedition hervorragend geleistete Arbeit, bedanken wir uns recht herzlich bei allen Offizieren der Special Branch in Juba und Torit (Südsudan): Sir George Ruony (Brigadier von der Polizei, Torit, Südsudan), Lt. Conel Abrahm Gauar (Bantiy, Südsudan), Dr. John Chuol Dhol (MAF-GOSS, Juba, Süd Sudan), Dr. Keah Nyah (Bantiy, Südsudan), Dr. Tayb Adam Abysnina (Khartoum, Sudan), Sultan Alex Kok (Katire, EES, Südsudan), Mr. Gesmallah Beter Aburas (Katire, EES, Südsudan), Ms. Kalkidan (Addis Ababa, Äthiopien) und bei allen freundlichen und ehrenvollen Personen der Lango und Achuoli Stämme. Wir möchten Dr. Elsadig H. Salim (Chernovtsy, Ukraine/Khartoum, Sudan) für seine Hilfe bei der Expedition und als Übersetzer besonders danken. 


\section{Literatur}

Gibeaux, Ch. 1994: Faune de Madagascar 81 Insectes Lépidoptères Pterophoridae. - Paris: 176 S.; 316 Figs., Paris.

Kovtunovich, V. \& Ustjuzhanin, P. 2011: On the fauna of the plume moths (Lepidoptera: Pterophoridae) of Lesotho. - African invertebrates. (derzeit im Druck)

\section{Anschrift der Verfasser:}

Ernst Arenberger

Naturhistorisches Museum Wien

Burgring 7 - 9

1010 Wien

Österreich/Austria

VLADIMIR GURKO

Chapaeva str. $43 \mathrm{~A} / 40$

58022, Chernovtsy

Ukraine

\section{Subject Editor:}

Dr. R. GAEDiKe
Prof. Dr. Samson A. Binyason

Ministry of Agricultural and Forestry (MAF)

GOSS, Juba

Southern Sudan 


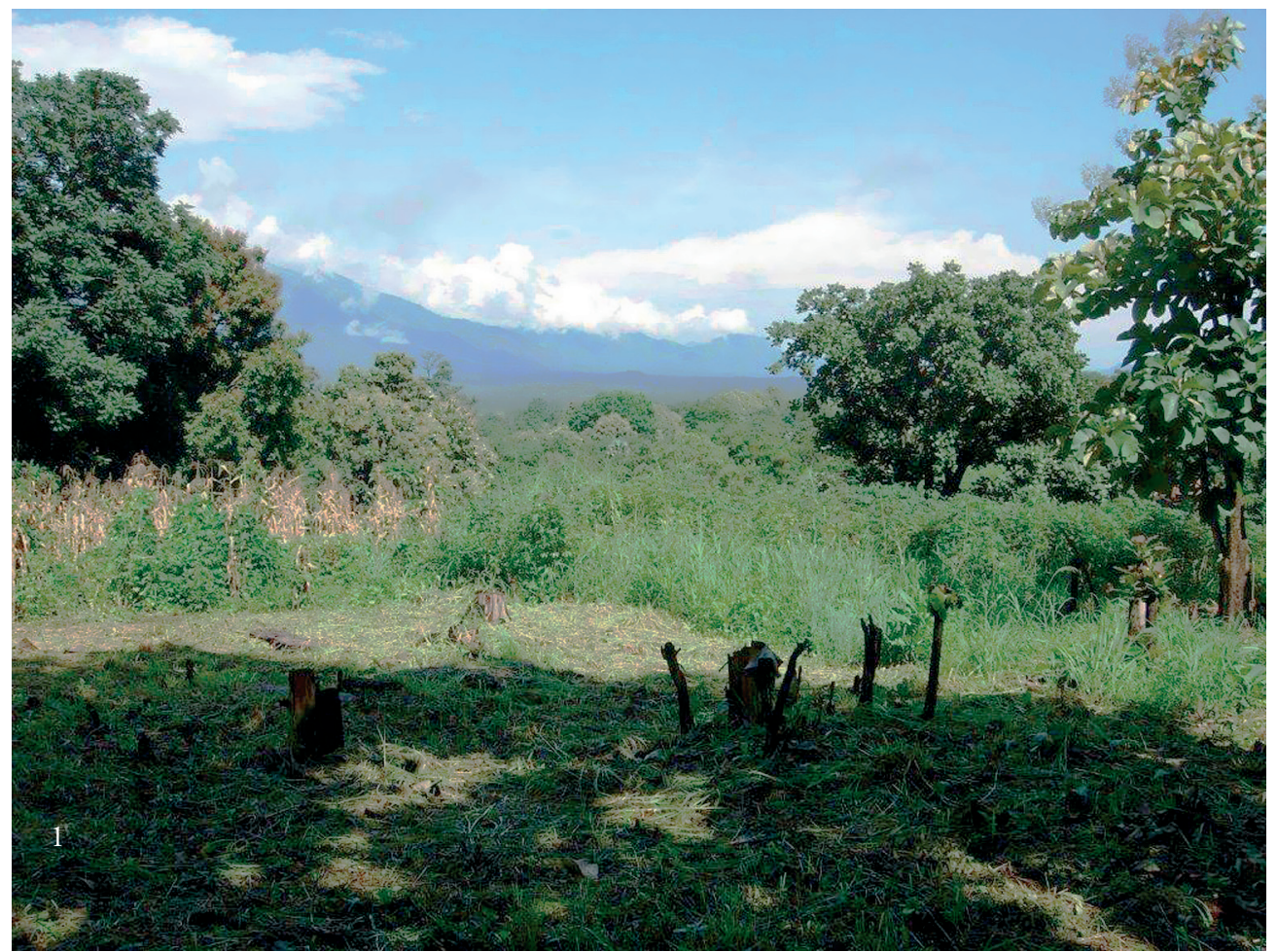

Fig. 1: Leuchtplatz in den Lolibai Mts.

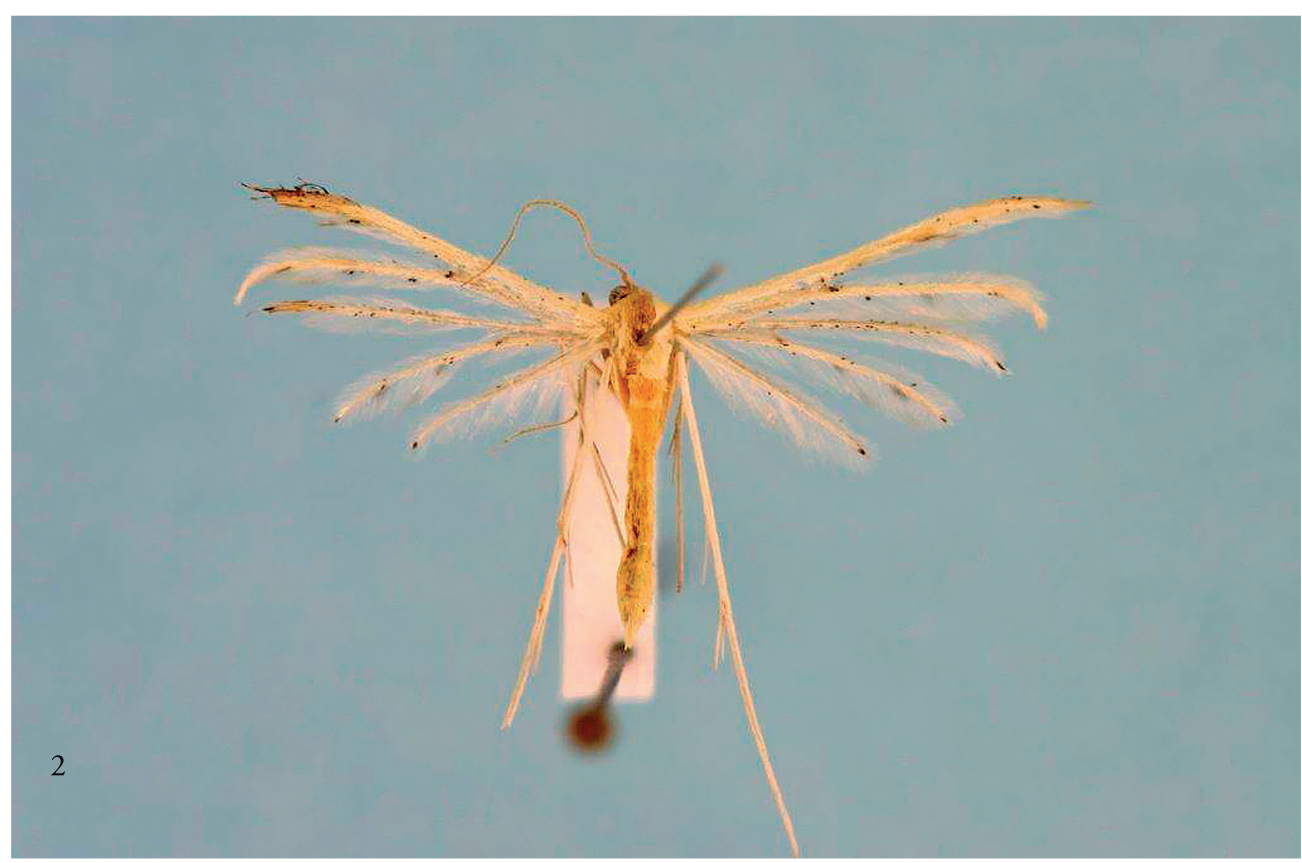

Fig. 2: Pterophorus albidus (Zeller, 1852). 

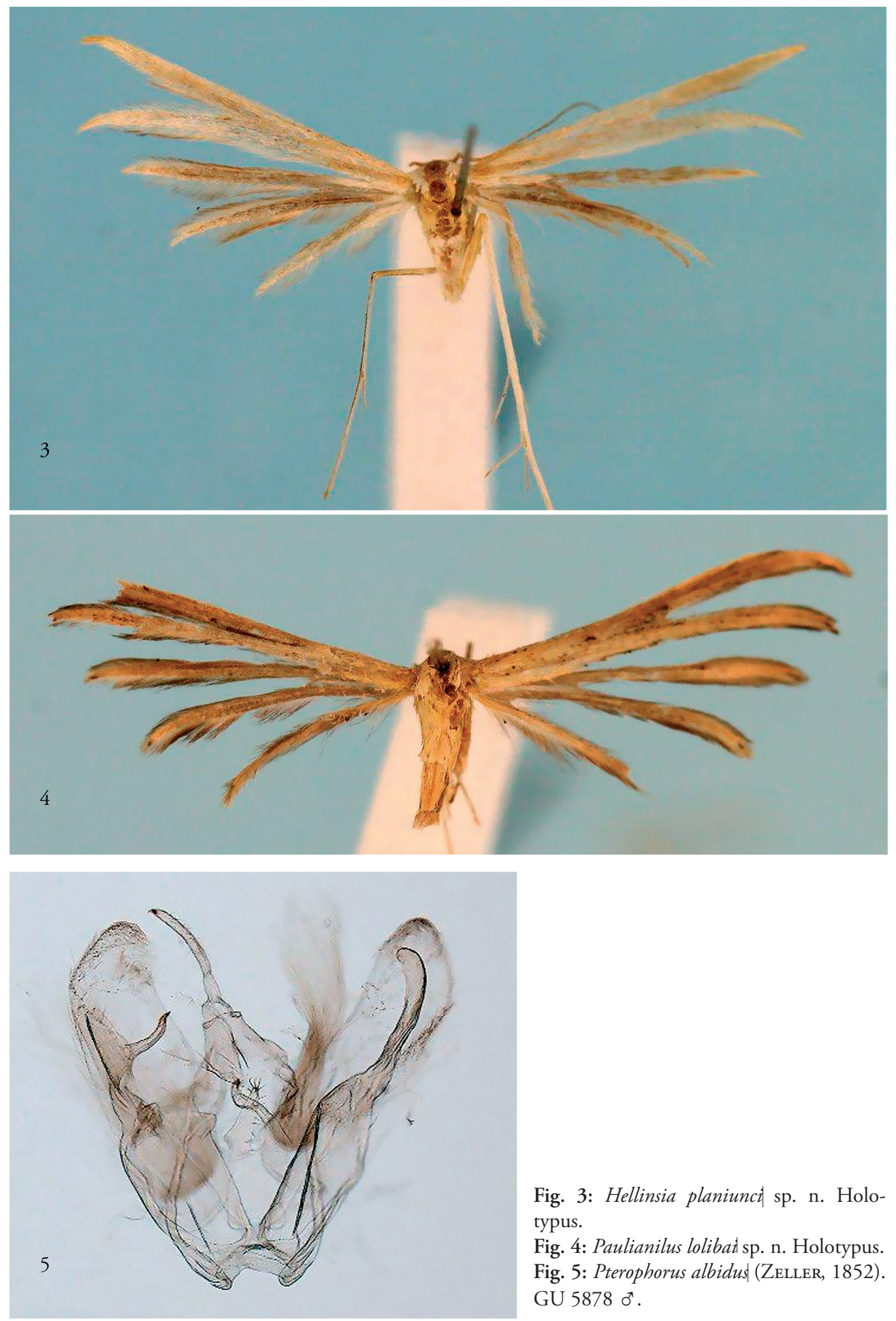

Fig. 3: Hellinsia planiunci sp. n. Holotypus.

Fig. 4: Paulianilus lolibai sp. n. Holotypus. Fig. 5: Pterophorus albidus (Zeller, 1852). GU $58780^{*}$. 


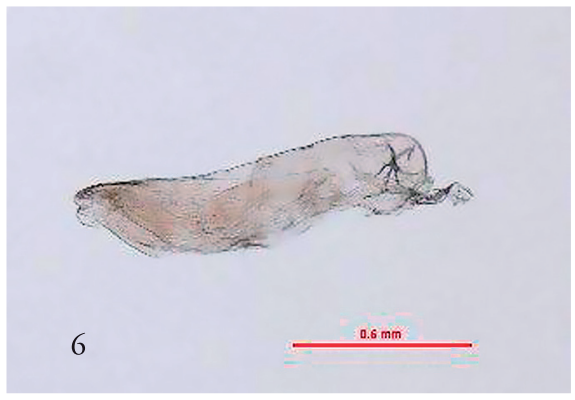

Fig. 6: dito, Aedoeagus (Maßstab 0,6 mm).

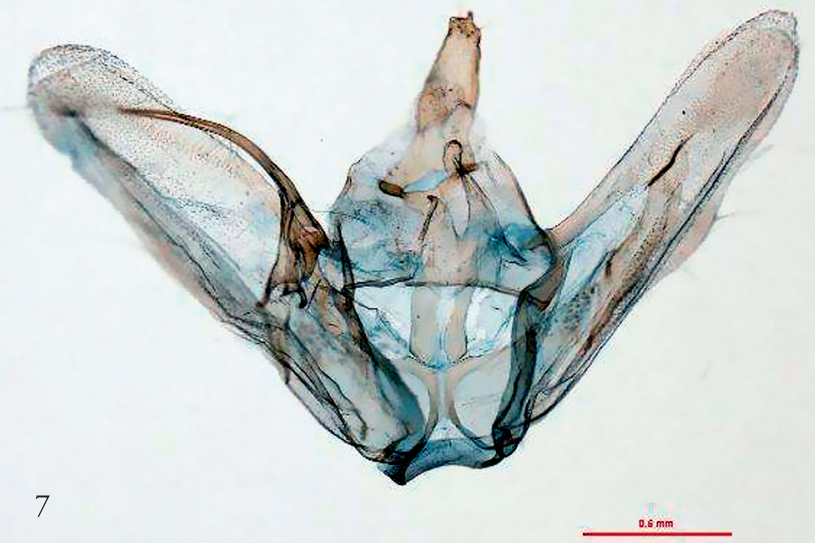

Fig. 7: Hellinsia planiunci sp. n. Holotypus. GU 5509 o (Maßstab 0,6 mm).

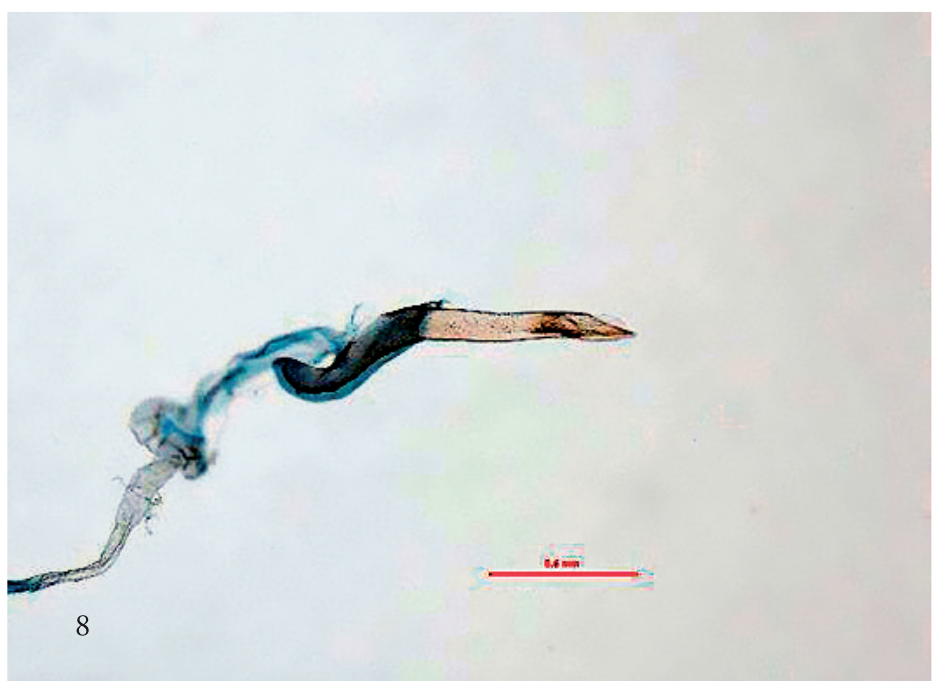

Fig. 8: dito, Aedoeagus (Maßstab 0,6 mm). 
9
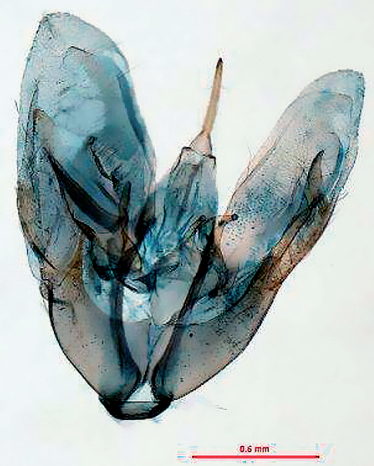

10
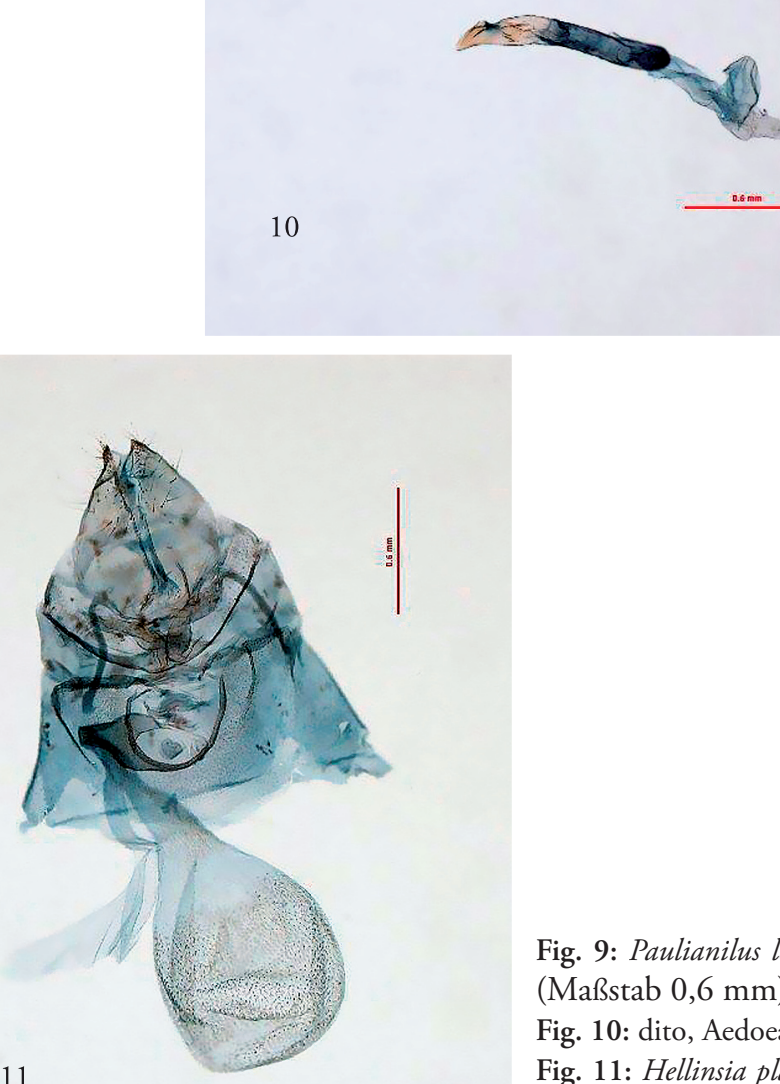

Fig. 9: Paulianilus lolibai sp. n. Holotypus. GU 5510 0 (Maßstab 0,6 mm).

Fig. 10: dito, Aedoeagus (Maßstab 0,6 mm).

Fig. 11: Hellinsia planiunci sp. n. Paratypus. GU 5511 우 (Maßstab 0,6 mm). 